\title{
Determinação de taninos e metilxantinas no guaraná em pó (Paullinia cupana Kunth, Sapindaceae) por cromatografia líquida de alta eficiência
}

\author{
Sandra A. Sousa, ${ }^{1}$ Suzana F. Alves, ${ }^{1}$ Joelma A. M de Paula, ${ }^{2}$ Tatiana S. Fiuza, ${ }^{3}$ \\ José R. Paula, ${ }^{1}$ Maria T. F. Bara ${ }^{*}, 1$
}

\author{
${ }^{1}$ Laboratório de Pesquisa em Produtos Naturais, Faculdade de Farmácia, Universidade Federal de Goiás, \\ Caixa Postal 131, 74605-220 Goiânia-GO, Brasil, \\ ${ }^{2}$ Curso de Farmácia, Unidade Universitária de Ciências Exatas e Tecnológicas, Universidade Estadual de Goiás, \\ Caixa Postal 459, 75000-000 Anápolis-GO, Brasil, \\ ${ }^{3}$ Laboratório de Biologia Celular, Instituto de Ciências Biológicas, Universidade Federal de Goiás, \\ Caixa Postal 131, 74001-970 Goiânia, GO, Brasil.
}

\begin{abstract}
RESUMO: As sementes de guaraná (Paullinia cupana Kunth, Sapindaceae) são utilizadas como estimulante e os principais constituintes químicos são as metilxantinas e os taninos, tais como catequina e epicatequina. Este trabalho teve por objetivo investigar um método por cromatografia líquida de alta eficiência (CLAE) para quantificar, simultaneamente, cafeína, teofilina, teobromina, catequina e epicatequina em pó de guaraná. Os valores obtidos foram comparados com os resultados encontrados no método espectrofotométrico da Farmacopéia Brasileira IV ed. Pelo método cromatográfico, os teores de metilxantinas e taninos totais foram de $4,04 \%$ e $1,48 \%$, respectivamente. Os teores de metilxantinas e taninos totais, obtidos pelo método espectrofotométrico, foram de $4,88 \%$ e $4,05 \%$, respectivamente. Acredita-se que, nas condições experimentais empregadas, o método espectrofotométrico não foi específico, quantificando outras substâncias fenólicas presentes no guaraná. Portanto, sugere-se o emprego desse método cromatográfico no controle de qualidade do guaraná em pó, por ser preciso, sensível, específico e rápido.
\end{abstract}

Unitermos: Controle de qualidade, planta medicinal, guaraná, cafeína, catequina, CLAE.

\begin{abstract}
Determination of tannins and methylxanthines in powdered guarana (Paullinia cupana Kunth, Sapindaceae) by high performance liquid chromatography". The seeds of Paullinia cupana Kunth, Sapindaceae, are used as a stimulant and the main chemical constituents are the methylxanthines and tannins, as catechin and epicatechin. This study aimed to investigate a method by high performance liquid chromatography (HPLC) to quantify both caffeine, theophylline, theobromine, catechin and epicatechin in powdered of $P$. cupana. The values obtained were compared with the results of the spectrophotometric method of the Brazilian Pharmacopoeia IV ed. For the chromatographic method, the levels of methylxanthines and total tannins were $4.04 \%$ and $1.48 \%$, respectively. The levels of methylxanthines and total tannins obtained by the spectrophotometric method, were $4.88 \%$ and $4.05 \%$, respectively. It is believed that, under our experimental conditions, the spectrophotometric method was not specific and quantified others phenolic substances present in the $P$. cupana. Therefore we suggest the use of this chromatographic method in quality control of $P$. cupana powder, to be accurate, sensitive, specific and fast.
\end{abstract}

Keywords: Quality control, medicinal plant, caffeine, catechin, HPLC.

\section{INTRODUÇÃO}

O guaraná, Paullinia cupana Kunth é uma planta da família Sapindaceae, originária da região Amazônica, a qual possui clima tropical, quente e úmido. É amplamente utilizado na medicina tradicional brasileira, sendo empregada em bebidas caseiras e industriais, como tônico e estimulante. A parte utilizada é a semente seca e 
levemente torrada (Santos \& Mello, 2007).

Dentre os principais constituintes químicos do guaraná encontram-se as metilxantinas (cafeína, teofilina e teobromina) e os taninos condensados, que são compostos por unidades monoméricas interligadas, sendo as principais a catequina e a epicatequina (Moraes et al., 2003; Heard et al., 2006; Ushirobira et al., 2007). Podem ser identificados também as procianidinas B2, B3 e B4, que são dímeros compostos por unidades flavan-3ol (Ushirobira et al., 2007).

A cafeína (1,3,7-trimetilxantina) exerce efeito estimulante sobre o sistema nervoso central, músculos cardíacos, sistema respiratório e secreção de ácido gástrico. Também é considerada como um diurético fraco e relaxante muscular. A teobromina (3,7-dimetilxantina) tem ação diurética e a teofilina (1,3-dimetilxantina) tem predominantemente efeito broncodilatador (Alves \& Bragagnolo, 2002; De Maria \& Moreira, 2007). As substâncias catéquicas presentes no guaraná demonstram atividade antioxidante, antiviral, bactericida, moluscicida e de inibição de algumas enzimas extracelulares (Santos \& Mello, 2007; Yamaguti-Sasaki et al., 2007).

Alguns métodos são descritos para a quantificação dos constituintes do guaraná, dentre eles encontra-se o ensaio espectrofotométrico para a determinação de metilxantinas e taninos totais, constante na Farmacopéia Brasileira (2003) e por Pelozo et al. (2008). Outros trabalhos empregam métodos como eletroforese capilar (Sombra et al., 2005; Kofink et al., 2007) e cromatografia líquida de alta eficiência (CLAE) para a quantificação de cafeína, teofilina, teobromina, catequina e epicatequina no pó dessa planta medicinal (Bempong \& Houghton, 1992; Carlson \& Thompson, 1998; Ushirobira et al., 2004a; Ushirobira et al., 2004b).

Este trabalho teve por finalidade investigar, simultaneamente, o teor de cinco marcadores do guaraná em pó por técnica cromatográfica (CLAE), a fim de obter um método analítico preciso, específico, rápido e sensível.

\section{MATERIAL E MÉTODOS}

\section{Amostra}

A amostra, sementes de guaraná em pó (Paullinia cupana Kunth., Sapindaceae) foi adquirida de fornecedor qualificado, Lote 519437, submetida à análise farmacognóstica conforme descrição da Farmacopéia Brasileira (2003) e submetida à reação de identificação dos marcadores por CLAE, realizada por comparação dos tempos de retenção com os de substâncias padrões (Sigma), confirmando a identidade do material utilizado.

\section{Método cromatográfico}

O método por cromatografia líquida de alta eficiência (CLAE) utilizado foi baseado no trabalho de Saito et al. (2006), empregado na determinação de epigalatocatequina, epicatequina, catequina e cafeína em amostras de chá verde (Camellia sinensis (L.) O. Kuntze). Porém, a extração dos marcadores foi realizada de forma idêntica à citada no ensaio espectrofotométrico (Farmacopéia Brasileira, 2003). Foi utilizado um cromatógrafo líquido de alta eficiência modelo ProStar marca VARIAN, equipado com injetor manual, detector UV-Vis modelo 320 e sistema de bombas binário modelo 210.

As condições cromatográficas foram as seguintes: coluna cromatográfica C18 (Varian), $250 \times 4,6 \mathrm{~mm}, 5 \mu \mathrm{m}$, modo de eluição isocrático, comprimento de onda de 280 $\mathrm{nm}$, sensibilidade do sistema de 0,5 , volume de injeção de $20 \mu \mathrm{L}$, a vazão da fase móvel foi de $1 \mathrm{~mL} / \mathrm{min}$ e a fase móvel composta por água: acetonitrila:metanol:acetato de etila:ácido acético (89:6:1:3:1), pH 3,5.

Os cálculos dos teores de cafeína, teofilina, teobromina, catequina e epicatequina foram realizados através da construção de curvas de calibração submetidas à regressão linear, utilizando concentrações de 0,25 a 1,25 $\mu \mathrm{g} / \mathrm{mL}$ para teobromina e teofilina, 14 a $70 \mu \mathrm{g} / \mathrm{mL}$ para cafeína e 2,4 a $12 \mu \mathrm{g} / \mathrm{mL}$, para catequina e epicatequina. As análises foram realizadas em triplicata.

\section{Método espectrofotométrico}

O método utilizado para determinação de metilxantinas (cafeína, teofilina e teobromina) e taninos totais foi o descrito pela Farmacopéia Brasileira (2003). Foi utilizado espectrofotômetro modelo Cary 50, marca Varian. O cálculo do teor das metilxantinas e taninos totais foi realizado através da construção de curvas de calibração submetidas à regressão linear, sendo empregados cafeína e pirogalol como padrões, respectivamente. A curva de cafeína foi realizada na concentração de 5 a $25 \mu \mathrm{g} / \mathrm{mL}$ e a curva de pirogalol, na concentração de 10 a $50 \mu \mathrm{g} / \mathrm{mL}$. As análises foram realizadas em triplicata.

\section{RESULTADOS E DISCUSSÃO}

Análises de controle de qualidade de matériasprimas vegetais e do fitoterápico são complexas, porém imprescindíveis para contribuir para a qualidade do medicamento (Ushirobira et al., 2004b; Bara et al., 2004; Bara et al., 2006; Chaves \& Costa 2008; Engel et al, 2008; Paula et al., 2008; Alvarenga et al., 2009; Bueno \& Bastos, 2009; Couto et al., 2009).

Inicialmente, visando investigar a autenticidade da amostra, foi feita a análise microscópica do pó de guaraná em estudo, sendo observados fragmentos de células do parênquima cotiledonar, de cor amarelada, isodiamétricas, grãos de amido isolados, com hilo central e células pétreas agrupadas ou isoladas. $O$ pó apresentou cor castanho-avermelhada Esses dados estão de acordo 
com as especificações da Farmacopéia Brasileira (2003).

A transformação de uma planta em medicamento deveser feita de maneira que se preservem as características químicas e farmacológicas do vegetal, garantindo a sua ação biológica, a segurança de utilização e a valorização de seu potencial terapêutico (Santos \& Mello, 2007). Realizou-se então um ensaio de identificação química do guaraná em pó e por comparação dos cromatogramas dos cinco marcadores e da matéria-prima estudada constatouse a presença das metilxantinas, catequina e epicatequina (Figuras 1 e 2). A não detecção dos dímeros de unidades flavan-3-ol (Ushirobira et al., 2007) poderia ser devido a baixa concentração destas substâncias na matéria-prima utilizada, nas condições experimentais empregadas.

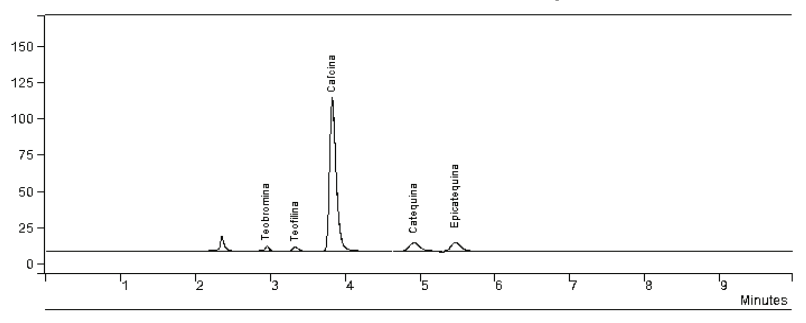

Figura 1. Cromatograma da solução padrão de cafeína $(3,82$ $\mathrm{min})$, teofilina $(3,32 \mathrm{~min})$, teobromina $(2,95 \mathrm{~min})$, catequina (4,93 min) e epicatequina (5,42 min). Condições cromatográficas: coluna C18 (Varian), 250 x 4,6 mm, $5 \mu \mathrm{m}$, comprimento de onda: $280 \mathrm{~nm}$, volume de injeção: $20 \mu \mathrm{L}$, vazão da fase móvel: $1 \mathrm{~mL} /$ min, fase móvel: água: acetonitrila:metanol:acetato de etila:ácido acético (89:6:1:3:1), pH 3,5

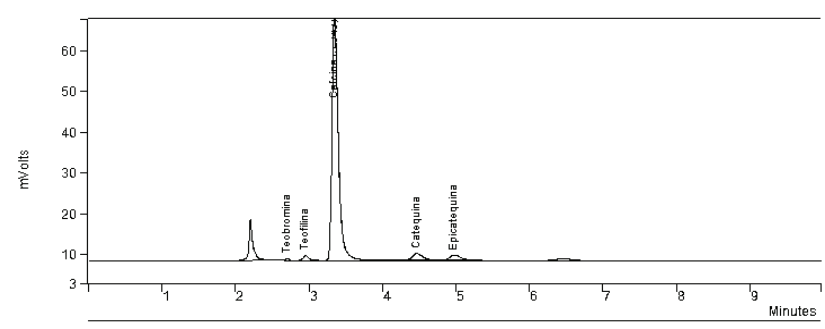

Figura 2. Cromatograma do guaraná em pó: teobromina $(2,66$ $\mathrm{min})$, teofilina $(2,91 \mathrm{~min})$, cafeína $(3,28 \mathrm{~min})$, catequina $(4,63$ $\mathrm{min})$ e epicatequina $(5,07 \mathrm{~min})$. Condições cromatográficas: coluna C18 (Varian), 250 x 4,6 mm, $5 \mu \mathrm{m}$, comprimento de onda: $280 \mathrm{~nm}$, volume de injeção: $20 \mu \mathrm{L}$, vazão da fase móvel: $1 \mathrm{~mL} /$ min, fase móvel: água:acetonitrila:metanol:acetato de etila:ácido acético (89:6:1:3:1), pH 3,5

Um método analítico empregado para a quantificação dos princípios ativos de um fitoterápico deve ser adequado ao fim ao qual se destina, tanto na etapa de extração dos analitos, quanto no doseamento dos marcadores com potencial efeito farmacológico. Nas análises de guaraná, a eletroforese capilar (Kofink et al., 2007) tem sido descrita como sendo um método capaz de identificar os enantiômeros naturais, $(+)$ - e (-)-catequina e
$(+)$ - e (-) epicatequina desta planta, o que foi confirmado pelo isolamento e purificação do flavan-3-ols e análises subseqüentes por LC-MS. Outros métodos como cromatografia líquida de alta eficiência (CLAE) são usados para a determinação de seus marcadores (metilxantinas e taninos) (Carlson \& Thompson, 1998; Sombra et al., 2005; Heard et al., 2006). Ensaios prévios realizados empregando as condições cromatográficas utilizadas na determinação de metilxantinas e catequinas em preparações contendo guaraná (Carlson \& Thompson, 1998) e para a avaliação das metilxantinas e catequinas liberadas de adesivos transdérmicos (Heard et al., 2006), usando como fase móvel tampão fosfato de potássio $0,25 \mathrm{M} \mathrm{pH} 3,5$ e metanol (77:23 v/v), em eluição isocrática, vazão de $0,9 \mathrm{~mL} / \mathrm{min}$, coluna C18 250 x 4,6 mm, $5 \mu \mathrm{m}$, detector UV a $272 \mathrm{~nm}$, foram considerados inadequados, uma vez que o tampão fosfato deve ser empregado em valores de $\mathrm{pH}$ entre 5,8 a 8,0 (USP 30, 2007). Outra condição cromatográfica foi investigada nesse estudo, como a citada para quantificação dos princípios ativos do guaraná (Sombra et al., 2005) e das metilxantinas da erva mate (Ilex paraguariensis) (Gnoatto et al., 2007), cuja fase móvel foi metanol e água $\mathrm{pH} 3,5$, na proporção 70:30, coluna $\mathrm{C} 18250$ x 4,6 mm e comprimento de onda $254 \mathrm{~nm}$, porém não houve boa separação dos picos da cafeína e catequina na análise do guaraná.

Um outro aspecto analítico importante é a escolha do $\mathrm{pH}$ da fase móvel que deve ser baseada no $\mathrm{pKa}$ das substâncias a serem eluídas. O pKa das metilxantinas, pode variar de 8,4 à 13,9 e das catequinas de 8,6 a 13,2 (Gennaro, 1999). O pH da fase móvel deve ser abaixo da constante de ionização, pois o aumento da forma ionizada do analito aumenta sua dissolução na fase aquosa e reduz seu tempo de retenção, já que a forma iônica passa através da coluna sem retenção (Ivanovic et al., 1995). O tamponamento é feito visando evitar variações de $\mathrm{pH}$, visto que quando as metilxantinas e catequinas são expostas a $\mathrm{pH}$ superiores a 4,0 são protonadas e assim, não interagem com a fase estacionária, sendo eluídas sem sofrer detecção (Aragão et al., 2005).

O teor de metilxantinas obtido pelo método espectrofotométrico foi de $4,88 \%$ e pelo método cromatográfico obteve-se uma concentração total de metilxantinas de 4,04\% (Tabela 1). Esses dados permitem sugerir que a maior concentração de metilxantinas obtida por espectrofotometria seja devido ao fato de que no comprimento de onda de $271 \mathrm{~nm}$, descrito na monografia da farmacopéia, são absorvidas outras substâncias (interferentes) como, por exemplo, as catequinas também presentes no pó de guaraná (Carlson \& Thompson, 1998), portanto, não apresenta especificidade. Além disso, devese ressaltar a vantagem da quantificação, individualmente, da teobromina e teofilina por CLAE, devido à sensibilidade dos métodos cromatográficos.

O método espectrofotométrico com absorção na região do UV, para análise de metilxantinas, além de apresentar precisão e exatidão, é um método barato e 
simples, porém é incapaz de distinguir as três metilxantinas do guaraná (De Maria \& Moreira, 2007).

Tabela 1. Comparação dos percentuais de teobromina, teofilina, cafeína, catequina e epicatequina em guaraná em pó por espectrofotometria e CLAE.

\begin{tabular}{cccc}
\hline Marcador & Classe & $\begin{array}{c}\text { Método } \\
\text { espectrofotométrico } \\
\text { Média* }(\%)\end{array}$ & $\begin{array}{c}\text { Método } \\
\text { cromatográfico } \\
\text { Média** }(\%)\end{array}$ \\
\hline $\begin{array}{c}\text { Teobromina } \\
\text { Teofilina }\end{array}$ & Metilxantinas & 4,88 & 0,03 \\
Cafeína & & & 0,06 \\
& & & 3,95 \\
Catequina & Taninos & 4,05 & Total: 4,04 \\
Epicatequina & & & 0,87 \\
& & & 0,61 \\
\hline
\end{tabular}

O teor de taninos totais encontrado pelo método espectrofotométrico foi de $4,05 \%$ e pelo método cromatográfico foram determinadas catequina e epicatequina, totalizando uma percentagem de $1,48 \%$ (Tabela 1). Como a extração realizada em ambos os métodos foi a mesma, ou seja banho-maria sob refluxo, na temperatura de $90{ }^{\circ} \mathrm{C}$, por $30 \mathrm{~min}$, acredita-se que pelo método espectrofotométrico são quantificadas fenóis totais presentes no guaraná, no comprimento de onda de $691 \mathrm{~nm}$, após reação com ácido fosfotúngstico SR em meio alcalino. A menor concentração destes compostos obtida por cromatografia líquida (comprimento de onda de $280 \mathrm{~nm}$ ) reforça a importância de emprego de métodos específicos na análise de fitoterápicos. Ressalta-se que mesmo não tendo sido quantificadas as procianidinas nas condições experimentais empregadas, a discrepância de 2,74 vezes a menos na concentração obtida por CLAE permite indicar o uso desse método.

Outros aspectos relativos à comparação dos procedimentos empregados nesse estudo frente à análise de taninos pela espectrofotometria de absorção na região da luz visível (691 nm), é que ele contém três etapas no processo de extração dos princípios ativos a serem quantificados (polifenóis totais e não adsorvidos pelo pó-de-pele), obtendo-se o percentual de taninos de forma indireta, além disso, a reação de cor utilizada para a medida das absorvâncias, não possui estabilidade (3 min para a leitura). Numa análise de rotina de controle de qualidade, ensaios simples (preparo da amostra em uma única etapa), sensíveis e específicos são mais vantajosos. Sendo assim, o método por CLAE seria mais indicado, além de permitir a quantificação dos cinco marcadores separadamente, numa única corrida cromatográfica (Alves \& Bragagnolo, 2002).

O método cromatográfico empregado nesse estudo apresentou precisão por repetibilidade $(n=6)$, com DPR menor que 5\% (Tabela 1) e linearidade para os cinco marcadores, com valores de $\mathrm{r}^{2}=0.997$ para teobromina $(\mathrm{y}=49813 \mathrm{x}-574,2), \quad 0,999$ para teofilina $(\mathrm{y}=54073 \mathrm{x}-$ $1379,5), 0.996$ para cafeína $(\mathrm{y}=46725 \mathrm{x}-11510), 0.998$ para catequina $(y=9355 x-1047,1)$ e 0,997 para epicatequina $(y=9731 x-839.8)$. A valores de DPR e $\mathrm{r}^{2}$ estão de acordo com a Resolução RE no 899, de 29 de maio de 2003 (Anvisa, 2003).

Deve-se destacar que para fins de garantia da qualidade de fitoterápicos, alguns atributos desejáveis são a autenticidade e a pureza. Autenticidade refere-se a provar que o material é verdadeiro e envolve parâmetros, incluindo análises químicas. Pureza diz respeito à avaliação de que não há material adulterante presente na matéria prima (Yadav \& Dixit, 2008). Além disso, visando maximizar as oportunidades e superar os desafios do mercado de fitoterápicos é essencial a padronização de matérias primas (Liu \& Wang, 2008), o que pode ser alcançado de forma mais consistente com o uso de métodos cromatográficos

\section{CONCLUSÃO}

O método cromatográfico utilizado para a determinação dos marcadores do guaraná em pó mostrou ser simples, rápido, preciso, linear, sensível e específico, sendo capaz de separar, identificar e quantificar simultaneamente cafeína, teofilina, teobromina catequina e epicatequina. Mediante os resultados obtidos pode-se sugerir o seu emprego no controle de qualidade de guaraná em pó.

\section{REFERÊNCIAS BIBLIOGRÁFICAS}

Alvarenga FCR, Garcia EF, Bastos EMAF, Grandi TSM, Duarte MGR 2009. Avaliação da qualidade de amostras comerciais de folhas e tinturas de guaco. Rev Bras Farmacog 19: 442-448.

Alves AB, Bragagnolo N 2002. Determinação simultânea de teobromina, teofilina e cafeína em chás por cromatografia líquida de alta eficiência. Rev Bras Cienc Farm 38: $237-$ 243.

Anvisa 2003. RE nº 899, de 29 de maio de 2003. Determina a publicação do "Guia para validação de métodos analíticos e bioanalíticos". Disponível em: http://www.anvisa.gov. br. Acesso em junho 2008.

Aragão NM, Veloso MCC, Bispo MS, Ferreira SLC, Andrade JB 2005. Multivariate optimisation of the experimental conditions for determination of three methylxanthines by reversed-phase high-performance liquid chromatography. Talanta 67: 1007-1013.

Bara MTF, Cirilo HN, Oliveira V 2004. Determinação de ginkgoflavonóides por cromatografia líquida de alta eficiência em matérias-primas e produtos acabados. Rev Eletronica Farm 1: 1-7.

Bara MTF, Ribeiro PAM, Arantes MCB, Amorim LLSS, Paula JR 2006. Determinação do teor de princípios ativos em matérias-primas vegetais. Rev Bras Farmacogn 16: 211215.

Bempong DK, Houghton PJ 1992. Dissolution and absortion of caffeine from guaraná. J Pharm Pharmacol 44: 769771.

Bueno PCP, Bastos JK 2009 A validated capillary gas chromatography method for guaco (Mikania glomerata S.) quality control and rastreability: from plant biomass to phytomedicines. Rev Bras Farmacogn 19: 218-223. 
Carlson M, Thompson RD 1998. Liquid chromatographic determination of methylxanthines and catechins in herbal preparations containing guaraná. J AOAC Int 81: 691701.

Chaves JS, Costa FB 2008. A proposal for the quality control of Tanacetum parthenium (feverfew) and its hydroalcoholic extract. Rev Bras Farmacogn 18: 360-366.

Couto RO, Valgas AB, Bara MTF, Paula JR 2009 Caracterização físico-química do pó das folhas de Eugenia dysenterica DC (Myrtaceae) Rev Eletronica Farm 6: 59-69.

De Maria CAB, Moreira RFA 2007. Cafeína: revisão sobre métodos de análise. Quim Nova 30: 99-105.

Engel IC, Ferreira RA, Cechinel-Filho V, Meyre-Silva C 2008. Controle de qualidade de drogas vegetais a base de Bauhinia forficata Link (Fabaceae) Rev Bras Farmacogn 18: 258-264.

Farmacopéia Brasileira 2003. 4 ed. São Paulo: Editora Atheneu, parte II, quinto fascículo.

Gennaro AR 1999. Remington Farmácia. 19 ed., vol. 2, Buenos Aires: Editorial Médica Panamericana.

Gnoatto SCB, Bassani VL, Coelho CG, Schenkel EP 2007. Influência do método de extração nos teores de metilxantinas em erva-mate (Ilex paraguariensis A. ST.Hil., Aquifoliaceae). Quim Nova 30: 304-307.

Heard CM, Johnson S, Moss G, Thomas CP 2006. In vitro transdermal delivery of caffeine, theobromine, theophylline and catechin from extract of guarana, Paullinia cupana. Int J Pharmac 317: 26-31.

Ivanovic D, Medenica M, Nivaud-Guernet E, Guernet M 1995. Effect of $\mathrm{pH}$ on the retention behaviour of some preservatives-antioxidants in reversed-phase highperformance liquid chromatography. Chromatographia 40: 652-656.

Kofink M, Papagiannopoulos M, Galensa R 2007 Enantioseparation of catechin and epicatechin in plant food by chiral capillary electrophoresis. Eur Food Res Technol 225: 569-577.

Liu Y, Wang MW 2008. Botanical drugs: challenges and opportunities. Life Sci 82: 445-449.

Moraes ML, Micke GA, Tavares MFM 2003. Separação e análise de metilxantinas em extratos de guaraná e erva-mate por eletroforese capilar. Rev Analytica 5: 44-50.

Paula JAM, Paula JR, Bara MTF, Rezende MH, Ferreira HD 2008. Estudo farmacognóstico das folhas de Pimenta pseudocaryophyllus (Gomes) L.R. Landrum - Myrtaceae. Rev Bras Farmacogn 18: 265-278.

Pelozo MIG, Cardoso MLC, Mello JCP 2008 Spectrophotometric determination of tannins and caffeine in preparations from Paullinia cupana var. sorbilis. Braz Arch Biol Technol 51: 447-451.

Saito ST, Welzel A, Suyenaga ES, Bueno F 2006. A method for fast determination of epigallocatechin gallate (EGCG), epicatechin (EC), catechin (C) and caffeine (CAF) in green tea using HPLC. Cienc Tecnol Aliment 26: 394400.

Santos SC, Mello JCP 2007. Taninos. In: Simões CMO (org.). Farmacognosia da planta ao medicamento. 5. ed. Porto Alegre: Editora da Universidade Federal do Rio Grande do Sul, p. 615-656.

Sombra LL, Gómez MR, Olsina R, Martínez LD, Silva MF 2005. Comparative study between capillary electrophoresis and high performance liquid chromatography in 'guarana' based phytopharmaceuticals. J Pharmac Biomed Anal
36: 989-994.

Ushirobira TMA, Yamaguti E, Uemura LM, Audi EA, Mello JCP 2004a. Avaliação fisico-química de sementes de guaraná secas por diferentes métodos. Rev Bras Farmacogn 14: 15-20.

Ushirobira TMA, Yamaguti E, Uemura LM, Mello JCP 2004b. Controle de qualidade de amostras de Paullinia cupana H.B.K. var. sorbilis (Mart.) Ducke. Acta Farm Bonaerense 23: 383-386.

Ushirobira TMA, Yamaguti E, Uemura LM, Nakamura CV, Filho BPD, Mello JCP 2007. Chemical and microbiological study of extract from seeds of guaraná (Paullinia cupana var. sorbilis). Lat Am J Pharm 26: 5-9.

United States Pharmacopeia 2007. $30^{\text {th }}$ ed. Rockville: The United States Pharmacopeial Convention

Yadav NP, Dixit VK 2008 Recent approaches in herbal drug standardization. Int J Integr Biol 2: 195-203.

Yamaguti-Sasaki E, Ito LA, Canteli VCD, Ushirobira TMA, Ueda-Nakamura T, Filho BD, Nakamura CV, Mello JCP 2007. Antioxidant capacity and in vitro prevention of dental plaque formation by extracts and condensed tannins of Paullinia cupana. Molecules 12: 1950-1963. 